\title{
Numerical study on two-phase turbulent flow of blunt combustor with strongly swirling flow
}

\author{
AHELIBAI HEINATI ${ }^{1, a}$, ZHIQIANG LI ${ }^{2, b}$, GAN DONG ${ }^{3, c}$ *
}

${ }^{1}$ National Key Laboratory of Science and Technology on Aero-Engine Aero-thermodynamics, School of Energy Power Engineering, Beihang University, Beijing 100191, China

${ }^{2}$ National Key Laboratory of Science and Technology on Aero-Engine Aero-thermodynamics, School of Energy Power Engineering, Beihang University, Beijing 100191, China

${ }^{3}$ National Key Laboratory of Science and Technology on Aero-Engine Aero-thermodynamics, School of Energy Power Engineering, Beihang University, Beijing 100191, China

aahelbai@126.com, ${ }^{\mathrm{b}}$ lizhiqiang@buaa.edu.cn, ${ }^{\mathrm{a}} \mathrm{dg} 19881208 @ 126 . c o m$

Keywords: Swirl flow, combustion, blunt body, two-phase flow, vortex shedding

Abstract. This study sets double injections in the inlet of the flow field to study the new blunt body's law of the flow patterns and the combustion efficiency under the condition of the two-phase flow. Compared with the vortex shedding mechanism of the V-gutter blunt, the computational results show that the vortex shedding mechanism of the coupling combustion stabilizer is controlled by the vortex of the swirling flow. During one shedding period, the heat and mass can be enhanced by the vortex source which is brought by the vortex breaking. The vortex structure is complex, and the increasing number is advantageous to heat and mass transfer between fluids. Under the combustion condition, the violent chemical reactions behind the blunt body bate the vortex shedding process. The flow field and vortex becomes more symmetric. The velocity field and pressure distribution behind the blunt body tends to be uniformly distributed under the combustion condition. Kerosene particles and air will be mixed and burned more fully, which is the cause of the function of the cyclone.

\section{Numerical simulation of the two-phase V-gutter blunt body under cold condition}

\section{Geometrical structure}

This paper uses the Du Yiqing's experimental geometry size, who is from Huazhong University of Science and Technology. The experiment was conducted in an open wind tunnel. Experimental blunt body is placed in the horizontal axis of rectangular duct, cross section of Rectangular duct is $250 \mathrm{~mm}$ $\times 250 \mathrm{~mm}$, length is $1500 \mathrm{~mm}$, test section is $700 \mathrm{~mm}$.

Du Yiqing's experimental parameters for V-gutter:

Table $1 \mathrm{~V}$-gutter experimental parameters

\begin{tabular}{cc}
\hline Parameter & Number \\
\hline Diameter & $D=0.45 \mathrm{~m}$ \\
Inlet velocity & $U=30 \mathrm{~m} / \mathrm{s}$ \\
Reynolds number & $\mathrm{Re}=U D / \mathrm{v}=615872$ \\
Blockage ratio & $18 \%$ \\
Aspect ratio & 2.22 \\
Turbulence intensity & $1 \%$ \\
\hline
\end{tabular}




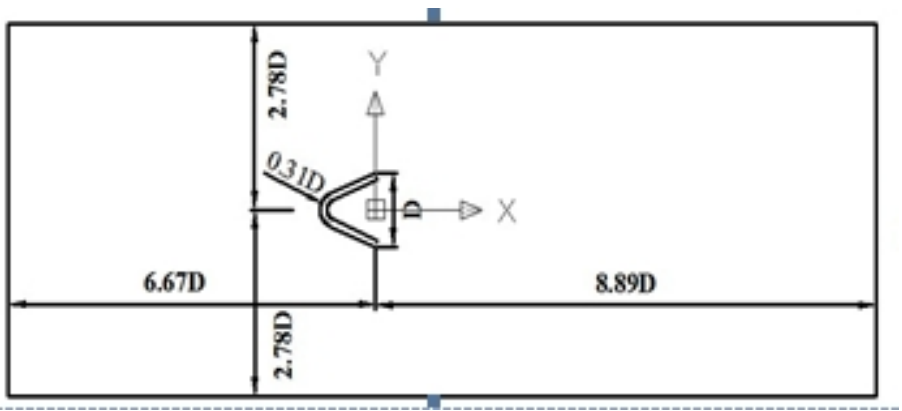

Fig. 1 V-gutter two dimensional cross section

As for blunt body combustor with strongly swirling flow, this paper is based on the above geometrical structure, via passing the swirl tube across the center of the V-gutter, it researches the vortex shedding law and flow field distribution of blunt body with strongly swirling flow. The diameter of the swirling tube is $10 \mathrm{~mm}$. Combined combustion stabilizer's geometry is shown in fig. 2 , swirl inlet is located in the symmetrical place to the swirl tube's XY-plane, these two swirl inlet produce swirl flow.

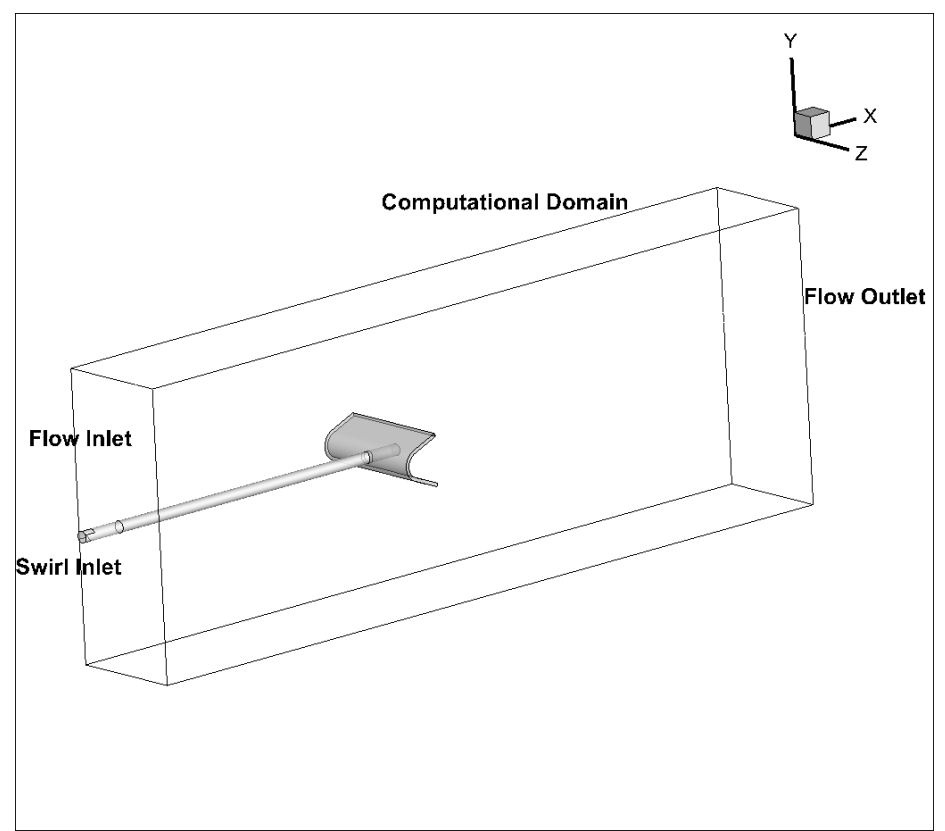

Fig. 2 combined structure of the V-gutter with strong swirl

\section{Boundary conditions}

The inlet flow is air under cold condition, velocity inlet with the value of $30 \mathrm{~m} / \mathrm{s}$, the outlet is pressure outlet , two sides are symmetry, computational domain's up wall and down wall are sliding wall; Liquid phase is $\mathrm{C} 12 \mathrm{H} 23$ (Kerosene), double injections in the inlet of the flow field are symmetrical to each other, the specific coordinate is $(-0.29, \pm 0.05,0)$, liquid-phase oil droplet diameter is $0.0001 \mathrm{~m}$, flow rate of each injection is $0.0004 \mathrm{~kg} / \mathrm{s}$.

\section{V-gutter's vortex shedding pattern with two phase flow in cold condition}

Fig. 3 shows the instantaneous flow line distribution in the central section of the V-gutter under cold two-phase flow condition, in which $\tau$ is a characteristic time of a complete cycle of vortex shedding. It can be known from the theory of correlation topological analysis of vortex stability [10], at a low Reynolds number, the existence of a two dimensional symmetric closed bubble separation vortex is unstable. Graph (a) shows, In vortex layer near to the edge of the blunt body, Kelvin-Helmholts instability phenomenon begins to appear, the front and rear position continues to produce the pulsation and the dislocation, and the size of the lower vortex is smaller than the size of the upper vortex, the large vortex scale formed by the shear layer instability shedding is basically equivalent to the diameter of the V-gutter blunt body. However, due to the strong vorticity transport 
function of the vortex itself, the large vortex scale further increases inevitably by gaining vorticity from the shear layer.

With the further development of the flow field, when $t=\tau / 3$ in fig. (b), it can be seen that upper large vortex moves away from blunt body to downstream, because it can't receive vorticity supplement from the blunt body surface shear layer, its scale gradually decreases with the effect of viscous dissipation, while the smaller vortex scale increases gradually by gaining energy from the blunt body surface shear layer. As time goes by, when $t=/ 2$ in fig. (c), a small vortex is reformed above the blunt body under the presence of shear, while the upper large vortex after shedding has been dissipated basically. On the contrary, below large vortex gains vorticity, with the dissipation of upper large vortex, the pressure reduces, the vortex layer is pulled into the tail flow and the shedding occurs.

When $t=2 \tau / 3$ in fig. (d), the large vortex beneath the blunt body transfers to downstream after shedding. During the process of transferring, the vorticity is constantly dissipated, at the same time, the newly formed small vortex above the blunt body begins to obtain the vorticity supplement from the wall shear layer and gradually increases, while the original vortex that sheds into the trail disappears after a constant dissipation;

When $\mathrm{t}=5 \tau / 6$ in fig. (e), after a certain time, the below large vortex after shedding has become smaller after constant dissipation, at the same time, the newly formed upper vortex has grown bigger after gaining vorticity supplement, and become unstable; While $t=\tau$ in fig. (f), below vortex which flows into wake disappear after constant dissipation, at this time, blunt body's bottom shear reproduce shear small vortex with effect of the shear stress, the upper vortex sheds, the new cycle begins.

Such cycled vortex shedding pattern behind the blunt body makes fluid form up a concentrated vortex behind blunt body, the vorticity sheds after reaching the maximum value and flows to the downstream of the flow field. At this time, a concentrated vortex after blunt body is reformed, such cycled vortex shedding mechanism determines the efficiency of the flame stabilization of the blunt body.

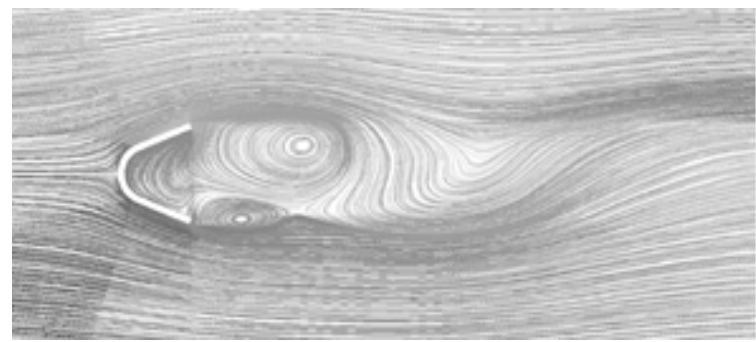

(a) $t=\tau / 6$

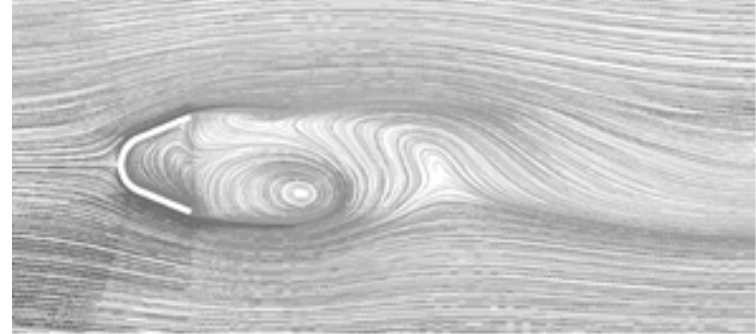

(c) $t=\tau / 2$

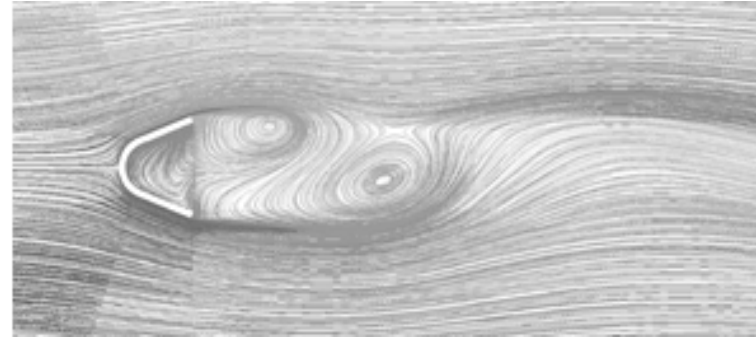

(e) $t=5 \tau / 6$

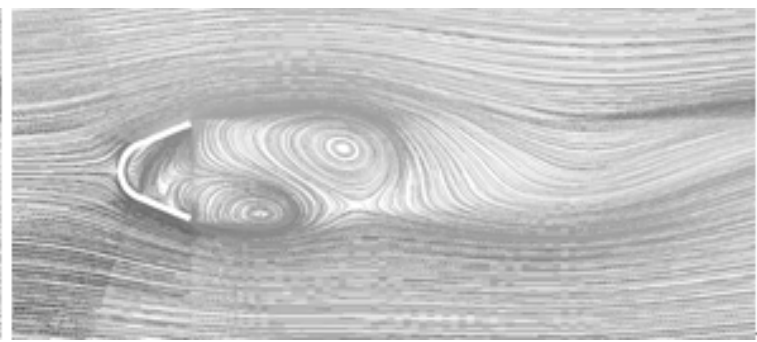

(b) $t=\tau / 3$

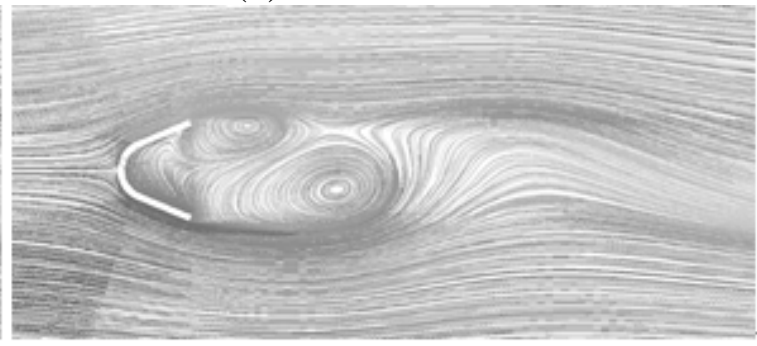

(d) $t=2 \tau / 3$

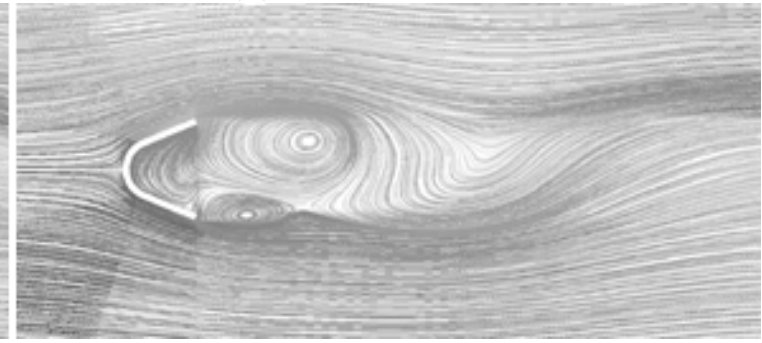

(f) $t=\tau$

Fig. 3 Instantaneous flow line in the central section of the V-gutter under cold two-phase flow condition 


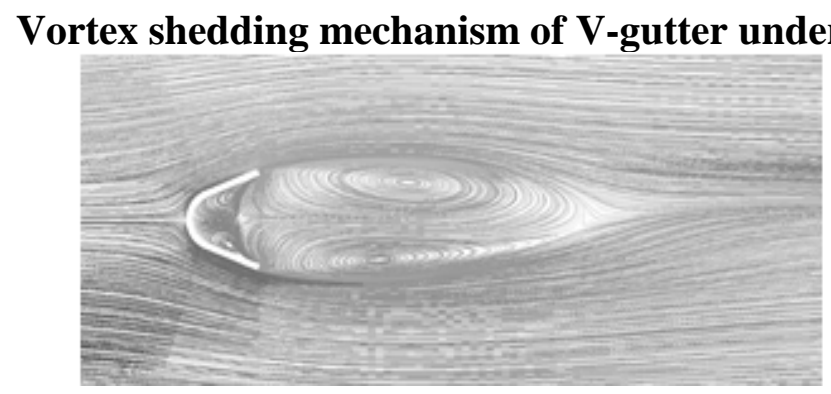

(a) $t=\tau / 4$

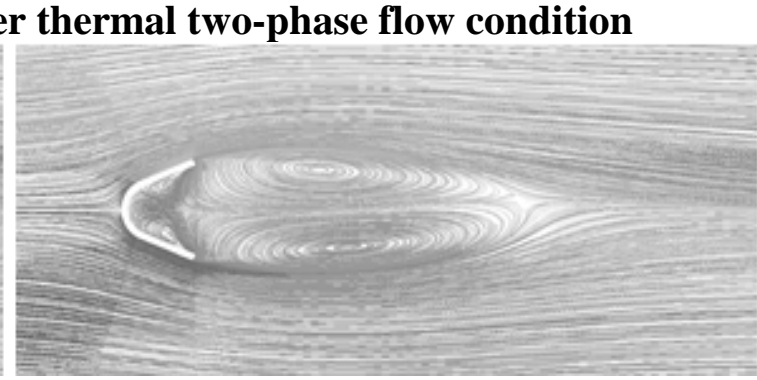

(b) $t=\tau / 2$

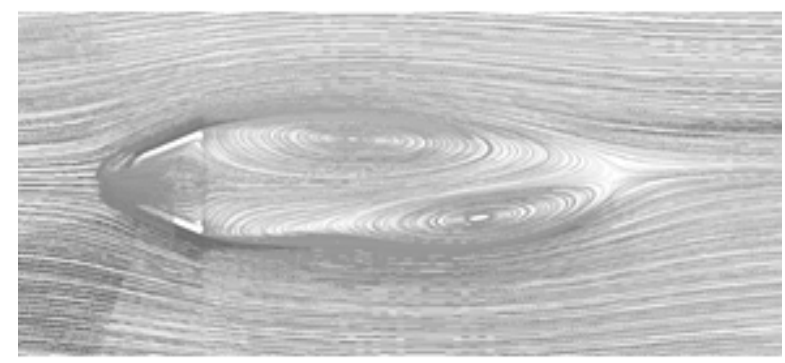

(c) $\mathrm{t}=3 \tau / 4$

Fig. 4 Central section's instantaneous flow line distribution of V-gutter with thermal state two-phase flow

Fig. 4 shows central section's instantaneous flow line distribution of V-gutter with thermal state two-phase flow. It can be seen that, compared to cold condition, the vortex is stretched obviously, vortex shedding period slows down suddenly, this is because violent chemical reaction weakens the effect of turbulence.

\section{Vortex structure}
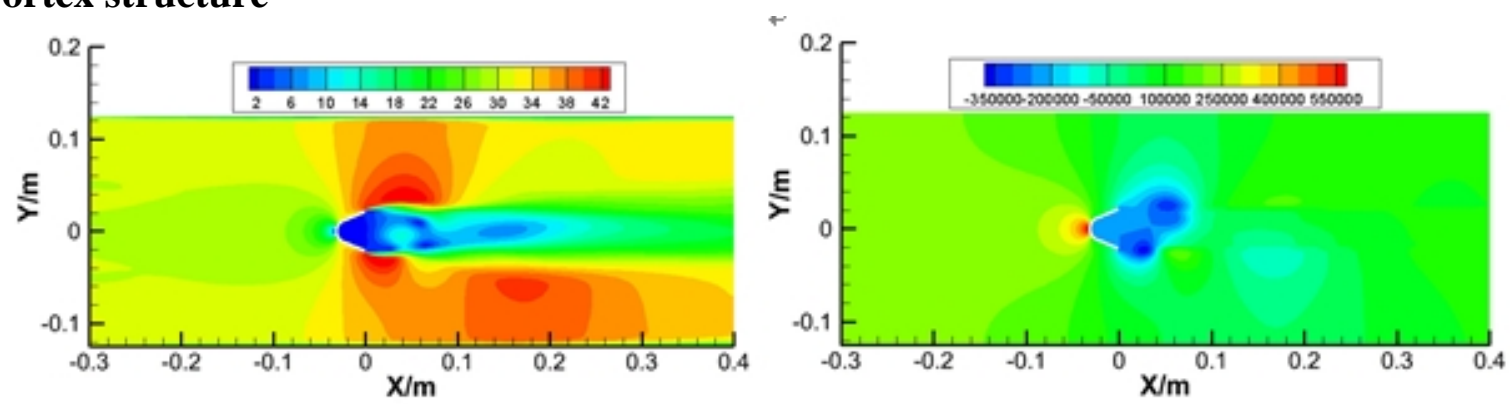

Fig. 5 Velocity and static pressure counters of the central section under cold condition

Under the cold condition, instantaneous velocity counter and static pressure cloud of V-shaped blunt body's central section are shown in fig.5. As is shown that we can approximately consider the low pressure zone in the above and beneath of blunt body as the vortex core of shear layer. The pressure distribution caused by the vortex shedding of upper and lower shear layer has asymmetric feature. From the velocity contour it can be seen clearly that, the vortex shedding from upper and lower shear layer is gradually dissipated as it flows to downstream. According to velocity, the most concentration of vorticity is located in upper and lower edge of the bluff body.
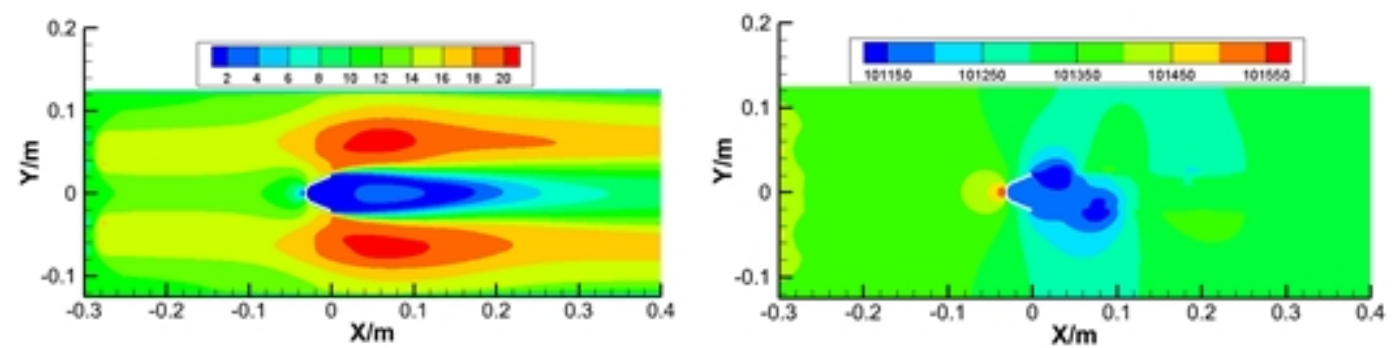

Fig. 6 Instantaneous velocity counter and static pressure cloud of the central section in thermal state 
Fig. 6 shows the instantaneous velocity counter and static pressure cloud of the central section of the V-shaped blunt body in thermal state. It can be seen that there exists injected fuel particles' moving track, due to the violent chemical reaction in the trailing edge of the blunt body, almost up and down symmetric high speed flow fields are formed, vortex is made to be more symmetrical; This is also confirmed from the pressure counter, pressure distributions at the behind of the blunt body in thermal state condition are basically symmetrical, which inhibits the vortex shedding because of the chemical reaction's inhibitory effect on the vortex shedding.

\section{Numerical simulation of the flow and combustion of the gutter-swirl combination}

\section{Geometrical Structure}

This section, still by passing swirl tube into the center of the V-shaped bluff body, researches vortex shedding patterns and the flow distribution features of the V-shaped bluff body combined with swirl. Swirl tube diameter is $10 \mathrm{~mm}$. The structure of combined combustion stabilizer is the same as in Figure 2, swirl tube inlet is located in the position which are symmetric to each other via the XY plane. Swirl flow begins from there.

\section{Boundary conditions}

Mainstream flow is air, temperature is $1000 \mathrm{~K}$, velocity inlet with the speed of $30 \mathrm{~m} / \mathrm{s}$, inlet turbulence intensity of $1 \%$, hydraulic diameter of $0.25 \mathrm{~m}$, pressure outlet, two symmetric sides, up and down walls of the domain are free slip wall. Liquid is $\mathrm{C} 12 \mathrm{H} 23$ (kerosene), nozzle position is near the inlet face, vertically symmetrical, specific coordinates $(-0.29, \pm 0.05,0)$, liquid droplet diameter is $0.0001 \mathrm{~m}$, droplet's temperature is $400 \mathrm{~K}$, each nozzle flow rate is $0.0004 \mathrm{~kg} / \mathrm{s}$.

\section{Thermal two-phase flow vortex structure of the gutter-swirl combination}

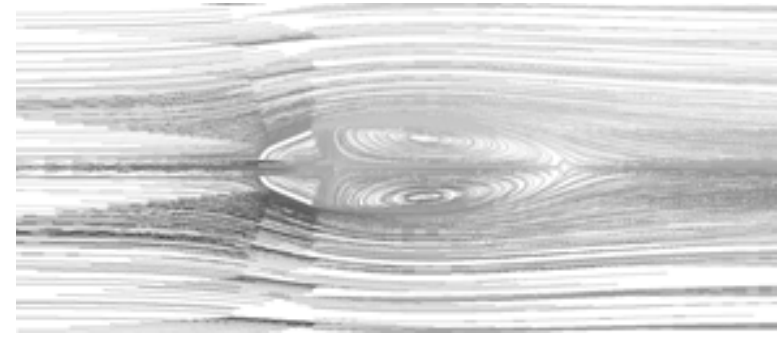

Fig. 7 two-phase flow streamlines of V-shaped bluff body-strong swirl combination

Figure 7 is two-phase flow streamlines of $\mathrm{V}$-shaped bluff body-strong swirl combined combustion stabilizer in thermal state. As can be seen, since there is a swirl passing into the center of the bluff body, it further promotes the burning process, vortex shedding process reaches to stop in recirculation zone, forming almost symmetrical vortex pairs, moreover, it further increases the size of the recirculation zone.
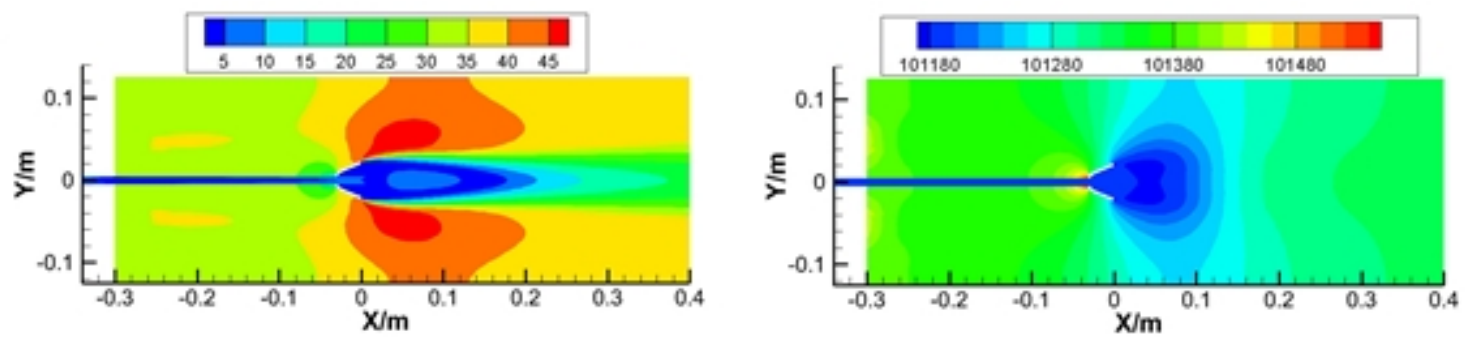

Fig. 8 Instantaneous static pressure contour and velocity counter of the central section of the V-gutter blunt body-swirl combination under two-phase thermal state.

Under thermal conditions, the instantaneous static pressure contour and velocity counter of the central plane of the combustion stabilizer with V-shaped-swirl combination are shown in Figure 8. It can be seen from the figure, the violent chemical reaction contributed to the high-speed flow field area 
vertically symmetrical to each other in the back of the combustion stabilizer, and the rear edge of the bluff body shear vortex pair are almost symmetric; The pressure counter also confirms this point. In thermal condition, the pressure distributions in the back of the blunt body are substantially symmetrical, this symmetrical pressure distribution suppresses vortex from shedding.

\section{Parameter Analysis}

\section{Resistance characteristics and vortex shedding period}

Drag coefficient is one of the important dimensionless parameter of blunt body resistance characteristics, its expression is:

$$
C_{D}=\frac{\overline{F_{D}}}{\frac{1}{2} \rho u^{2} A}
$$

Where: $\overline{F_{D}}$ is the average value of the blunt body's resistance to the fluid in the flow field, $u$ is flow velocity, A is size of the bluff body's drainage surface.

Pulsating drag coefficient is nondimensionalized by using RMS value of the pulsation resistance value, which can be calculated in the below equation:

$$
C_{D}^{r m s}=\frac{\sqrt{\left(F_{D}-\overline{F_{D}}\right)^{2}}}{\frac{1}{2} \rho u^{2} A}
$$

Where: $F_{D}$ is the fluid resistance received by the cylinder at a transient time.

One of the important dimensionless parameters characterizing the vortex shedding process is Strouhal number, defined as:

$$
\mathrm{St}=\mathrm{f} \mathrm{D} / \mathrm{u}
$$

Where: $\mathrm{f}$ is vortex shedding frequency of the flow around a blunt body, $\mathrm{D}$ is the diameter of the blunt body.

Table 2 shows the drag coefficient and vortex shedding period of the standard V-gutter and $\mathrm{V}$-shaped blunt body with swirl in two-phase thermal and cold conditions.

Table 2 Drag coefficient and vortex shedding period under different conditions

\begin{tabular}{ccccc}
\hline Blunt body type & $\begin{array}{c}\text { Chemical reaction } \\
\text { situation }\end{array}$ & $\overline{C_{D}}$ & $C_{D}^{\text {rms }}$ & St \\
\hline \multirow{2}{*}{ V-gutter } & non-reaction & 1.777 & $7.72 \times 10-3$ & 0.3439 \\
\cline { 2 - 5 } & reaction & 1.894 & $8.29 \times 10-2$ & 0.0449 \\
\hline \multirow{2}{*}{$\begin{array}{c}\text { V-gutter combined } \\
\text { with swirl }\end{array}$} & non-reaction & 1.3 & $3.04 \times 10-4$ & 0.2274 \\
\cline { 2 - 5 } & reaction & 1.44 & $3.68 \times 10-4$ & 0.0207 \\
\hline
\end{tabular}

As is shown from the table, chemical reaction can increase the pulsating drag coefficient and drag coefficient, in this table, after the reaction in the flow field of the two combustion stabilizers, pulsating drag coefficient and average drag coefficient were slightly increased; However, chemical reaction decreases the Strouhal number to the $10 \%$ of its value before reaction approximately, that is 
to say, vortex shedding period increases sharply, reflecting chemical reaction's inhibitory effect on turbulent flow; In addition, in cold condition, V-gutter-swirl combined combustion stabilizer's vortex shedding frequency approximately decreased 33\% compared to standard V-gutter.

\section{The average recirculation zone properties}

The average recirculation zone properties reflect the structural characteristics of the averaged vortex in the back of the blunt body. In cold condition, the bigger is the average recirculation zone, the better is the efficiency of the flame stabilization. Figure 9 respectively shows the average streamline distribution of the central section of the V-shaped bluff body and V-gutter with swirl combustion stabilizer.

By comparison, without swirl, the recirculation zone is relatively narrow. After passing the swirl into the flow field, due to the rotating disturbance of the swirl, the recirculation zone gets larger. In addition, there occur two small vortex structures located in upper and lower side of the standard V-shaped blunt body's internal area, with their induction, two focused vortex flow forward; However, there occurs so-called solid-core vortex structure in the surrounding of the outlet of the swirl tube in the gutter-swirl combined combustion stabilizer, low pressure area existing in the vortex central area[11] could sucks nearby fluid to enter into the two focused vortex. With the increase of the swirl intensity, the core of the focused vortex flows backward, and the size of the vortex becomes bigger. As a whole, those vortex structures' commonly existence actually enhances the mixing effectiveness between fluids, and it is very conductive to the combustion stabilization.

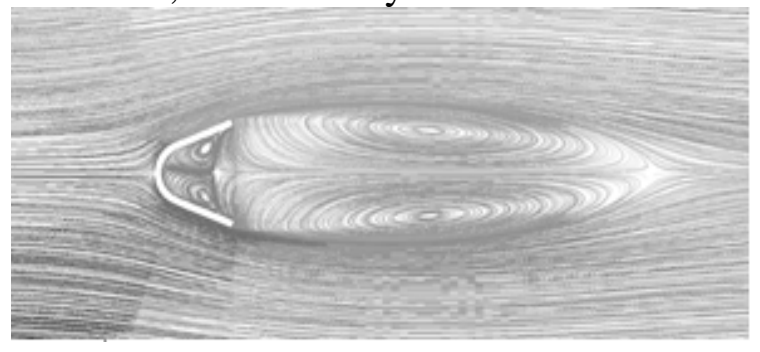

(a) $\mathrm{V}$

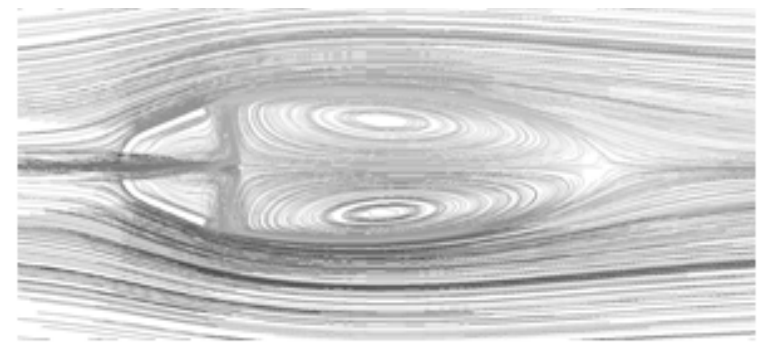

(b) $\mathrm{VX}$

Figure 9 stream line chart of the central section in two-phase burning state
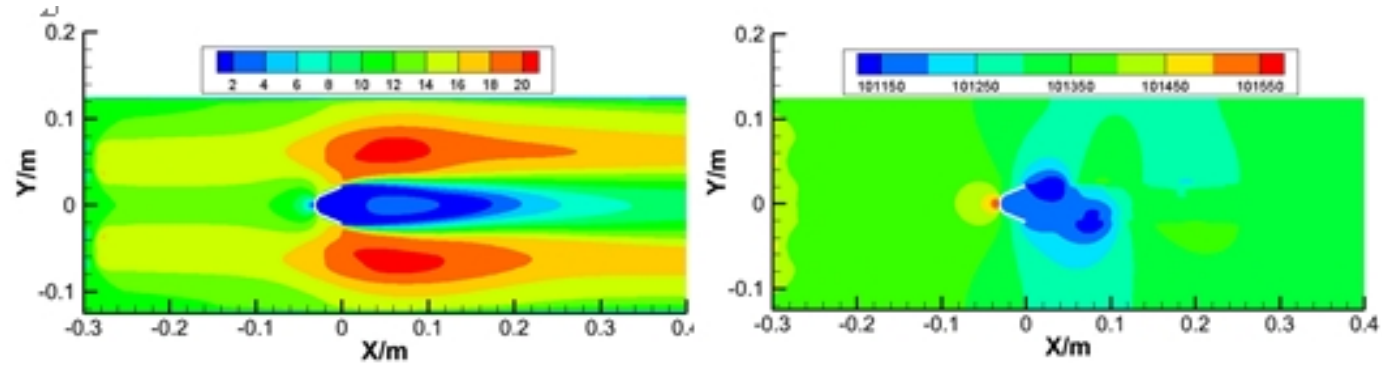

Fig. 10 Velocity and static pressure counters in the central section of the V-gutter under thermal state.
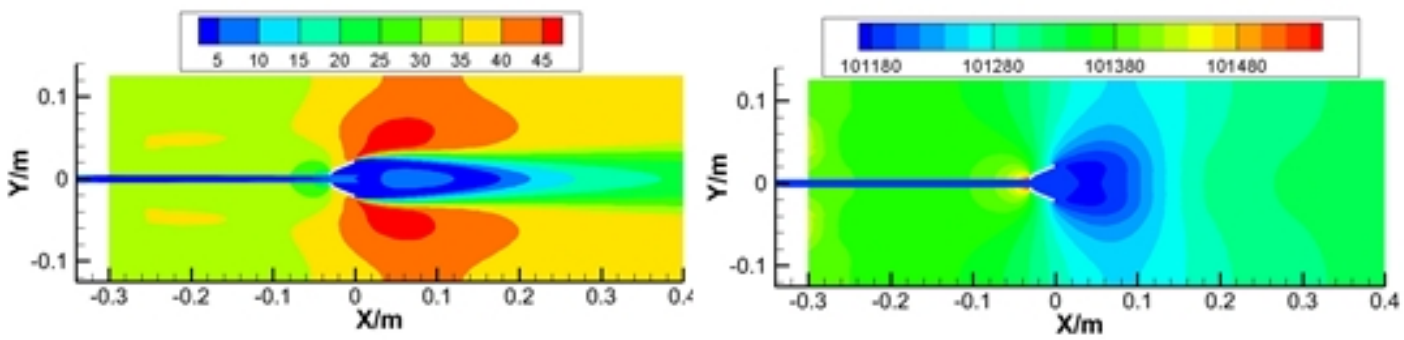

Fig. 11 Velocity and static pressure counters in central section of V-gutter and swirl combination under thermal state

It can be seen from the figure 10 and fig. 11 that there exists obvious trajectory of oil droplets in the flow field. With the effect of violent chemical reaction in the back of the blunt body, vertically 
symmetric high-speed flow fields are formed, stable vortexes are also symmetric; As is also proved by the figure of the pressure distribution

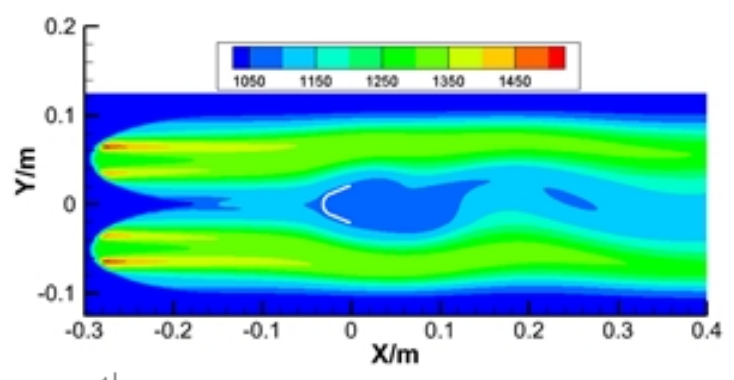

(a) V-gutter

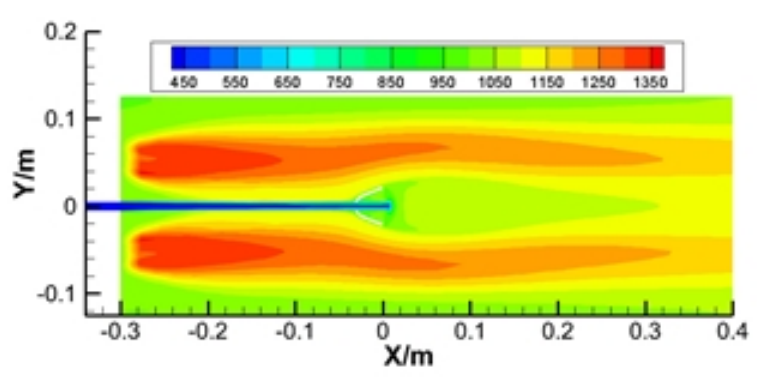

(b) V-gutter with swirl

Fig. 12 Temperature distribution counter in central section under thermal state

As for V-gutter, there still exists the vortex shedding phenomenon in two-phase burning flow field. While it almost disappears after using the swirl, burning efficiency is better.

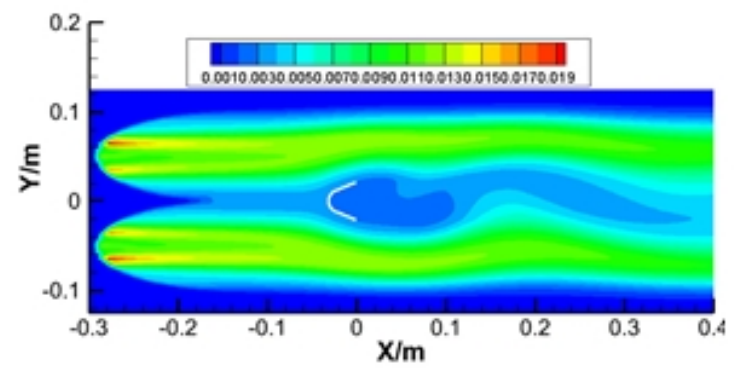

(a) V-gutter

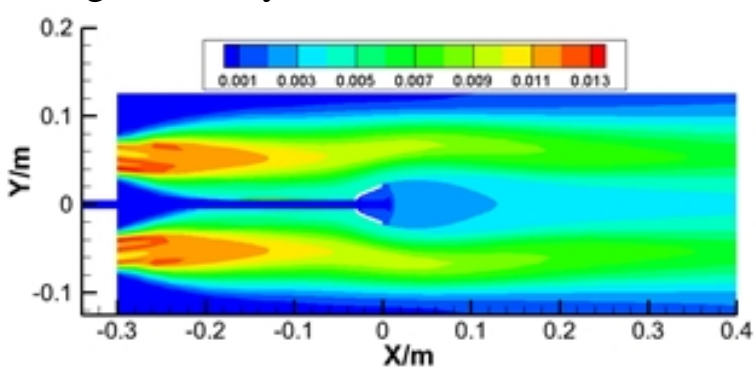

(b) V-gutter with swirl

Fig. 13 Water generation distribution counter of the central section of the chemical reaction

From fig. 13 it can be seen that, blunt body-swirl combination's flow field is more homogeneous, more conductive to the mixture of the oil and gas, completely burned high-temperature gas fills out reflux area. There is a mixed layer of mixed gas and high-temperature fuel-gas between recirculation zone and mainstream. When the fresh mixture gas mixing time and ignition induction period is less than the time of mixed gas shedding from the recirculation zone, flame remains stable.

\section{Conclusion}

Under cold condition, there still exists vortex shedding in two-phase flow field of flow around blunt body, and is mainly controlled by the shearing separated vortex which is produced by the alternate instability of up and down boundary layer of the blunt body; For the vortex shedding caused by the interaction and influence of swirl and blunt body, vortex shedding pattern is mainly controlled by the interaction of the swirl vortex generated by swirl and shear vortex caused by blunt body. Vortex structure is complex, the vortex number increases, which is conducive to heat and mass transfer between fluids.

Under thermal condition, there is an obvious trajectory of fuel oil droplets in the velocity field, due to the inhibition of the violent chemical reaction behind a bluff body to the vortex shedding, almost vertically symmetrical high-speed flow field area is formed, vortex pair becomes more symmetrical. In addition, under thermal condition, compared with the V-shaped bluff body, velocity and pressure distribution tend to be evenly distributed in the recirculation zone behind the blunt body in the flow field of the gutter-swirl combined combustion stabilizer, which is more conducive to the mixing and burning of the kerosene oil droplets with air, that is the beneficial role of swirl. 


\section{References}

[1] Ge Gao, Huang Ning. Theoretical and Experimental Research of the Dune Trapped Vortex[J]. Journal of Engineering Thermophysics, 1982, 3(1): 89-95.

[2] Maoling Yang.Zhong Quan,Xingyan Bai. EXPERIMENTAL INVESTIGATION ON EBMC FLAMEHOLDER [J]. Journal of Aerospace Power, 1998, 13(2): 185-188.

[3] Hongbin Zhang, Jigen Wang. DEVELOPMENT AND APPLICATION OF DOUBLE V TYPE FLAME STABILIZER [J]. Journal of Propulsion Technology, 1994, 15(1): 38-43.

[4]Qixiang Han, Hong Liu, Fang Wang. EXPERIMENTAL INVESTIGATION ON TOTAL PRESSURE LOSS OF VORTEX FLAME-HOLDER WITH HOLES [J]. Journal of Propulsion Technology, 1999, 20(4): 82-85.

[5]Hong Liu,Fang Wang,Jiahua Wang. Experimental Study of the Performanee of Special EvaPorating V-gutter Flame-Holder at Low Pressure [J]. Journal of Jiangsu Polytechnic University, 2004, 15(1):12-14.

[6]Fengdian Zheng, Huiqiang Zhang, Wenyi Lin. Experimental Investigation of Combustion Performance of U-Type Flame Holder [J]. Journal of Aerospace Power, 2003, 18(6): 808-812.

[7]Yiqing Du. Mechanism Investigation on Aircraft Afterburner Flameholder of High Efficiency、 Low Drag and High Temperature Resistance [D]. Wuhan: Huazhong University of Science and Technology, 2005.

[8]Eugene L, Caleb N C, John T C, et al. Novel carbureted flame holder for improved afterburner stability [R]. AIAA 2008-98, 2008.

[9]Jiezhi Wu, Huiyang Ma, Mingde Zhou. Vortex Mechanics[M]. Beijing: Higher Education Press, 1993.

[10]Jinhee Jeong, Fazie Hussain. On the identification of a vortex [J]. Fluid Mech. 1995, 285: 69-94.

[11]Lugt H. J. The dilemma of defining a vortex [J]. Fluid Mech., 1979, 309-321.

[12]Chongm S., Perrya. E. \& Cantwelbl. J. A general classification of three-dimensional flow field [J]. Phys. Fluids, 1990, A 2: 765.

[13]Metcalfer W., Hussainf, Menons \& Hayakawam. Coherent structures in a turbulent mixing layer: a comparison between numerical simulations and experiments [J]. Turbulent Shear Flows, 1985, 110. 